\title{
Melatonin ameliorates brain oxidative stress and upregulates senescence marker protein-30 and osteopontin in a rat model of vascular dementia
}

\author{
I Bin-Jaliah', HF Sakr ${ }^{2,3}$ \\ ${ }^{1}$ Department of Physiology, College of Medicine, King Khalid University, Abha, Saudi Arabia \\ ${ }^{2}$ Faculty of Medicine, Department of Medical Physiology, Mansoura University, Mansoura, Egypt \\ ${ }^{3}$ Faculty of Medicine and Health Sciences, Department of Medical Physiology, Sultan Qaboos University, \\ Muscat, Oman
}

Received: January 26, 2017

Accepted: January 16, 2018

\begin{abstract}
The aim of this study was to investigate the effect of melatonin on oxidative stress and senescence marker protein-30 (SMP30) as well as osteopontin (OPN) expression in the hippocampus of rats subjected to vascular dementia (VD). A total of 72 male rats were divided into six groups ( $n=12$ each) as follows: (i) untreated control (CON), (ii) shamoperated group, (iii) sham-operated + melatonin, (iv) rats exposed to VD induced by permanent bilateral occlusion of the common carotid arteries (BCCAO) leading to chronic cerebral hypoperfusion, (v) rats exposed to VD + melatonin, and (vi) rats exposed to VD + donepezil (DON). At the end of experiment, the hippocampal levels of acetylcholine (ACh), norepinephrine (NE), and dopamine (Dop) were measured. Expression of OPN was determined using immunohistochemistry, and SMP30 expression was determined using real-time PCR in the hippocampus. Hippocampal thiobarbituric acid reactive substances (TBARS) and total antioxidant capacity (TAC) were evaluated. The BCCAO group showed significantly decreased TAC $(p<0.05)$ and significantly increased in TBARS levels compared with the CON group. In addition, BCCAO significantly decreased $(p<0.05)$ the expression of both OPN and SMP30 and the levels of ACh, NE, and Dop in the hippocampus compared with CON treatment. Treatment with melatonin significantly increased OPN and SMP30 expression and ACh, NE, and Dop levels in the hippocampus with amelioration of the oxidative stress compared with BCCAO rats. Melatonin might produce a neuroprotective effect through its antioxidant action and by increasing the expression of SMP30 and OPN that is not comparable with that of DON.
\end{abstract}

Keywords: vascular dementia, osteopontin, senescence marker protein-30, melatonin, oxidative stress

\section{Introduction}

Chronic cerebral hypoperfusion (CHP) due to cerebrovascular disease (29) is usually associated with loss of cognitive ability, including memory, language, attention, and problem-solving ability, which makes it a serious medical, social, and economic burden for the society (4). Risk factors include hypertension, diabetes, generalized atherosclerosis, smoking, and heart diseases (29).

Melatonin (N-acetyl-5-methoxytryptamine) is a key endogenous indoleamine secreted by the pineal gland and other extrapineal tissues such as the retina. Aside from its critical role in regulating circadian rhythms, melatonin and its metabolites possess well-known

Corresponding author: Dr. Hussein F. Sakr

Faculty of Medicine, Department of Medical Physiology, Mansoura University

P.O. Box 35516, Mansoura, Egypt

Phone/Office: +20 50 2305557; Mobile: +20 11 27720093; Fax: +96824143514; E-mail: sakr_doctor@yahoo.com 
antioxidant, anti-inflammatory, and antiapoptotic properties in the central nervous system (CNS) $(11,25,26,34,56)$. These characteristics and the ability of melatonin to quickly and easily cross the blood-brain barrier made exogenous melatonin administration a drug of choice against aging (13) and some CNS conditions, such as Parkinson's disease (38), ischemia, and stroke $(33,35)$. To a large extent, these neuroprotective effects of melatonin are ascribed to its antioxidant and antiapoptotic functions and its role in protecting against mitochondrial dysfunction $(26,52,56)$.

Senescence marker protein-30 (SMP30) was first isolated as a hepatocyte aging factor in 1992. The expression of SMP30 was shown to decline with age. It is present in a variety of tissues, such as the brain, testes, and renal proximal tubular epithelial cells (19). The reported functions and activities of SMP30 are varied. Its major function is regulating cellular apoptosis through $\mathrm{Ca}^{2+}$ homeostasis $(19,41)$. SMP30 exerted antiapoptotic effects through either sustained and elevated cellular $\mathrm{Ca}^{2+}$ or Fas-mediated apoptosis (19). Moreover, SMP30-deficient brains showed increased Mac-1 protein and myeloperoxidase activity, suggesting a putative antioxidant action for SMP30 (46).

Various factors, such as Bcl-2, Bax, cytochrome c, p53, and Fas, have been linked to apoptosis pathways (12). Bcl-2 was first discovered in mammals as an antiapoptosis marker. If Bcl-2 levels are higher than those of Bax, the condition facilitates cell survival. On the other hand, when Bax is upregulated compared with Bcl-2, the dimer facilitates apoptosis and triggers caspase- 3 to cause a breakdown of cytoskeletal and nuclear proteins and nucleosomal fragmentation (22). Thus, the proportion of Bcl-2 relative to Bax may be a key controller of apoptosis.

Osteopontin (OPN) is a phosphorylated sialic acid-rich non-collagenous bone matrix protein that is distributed in various body fluids (18). OPN was shown to be an important factor in bone remodeling. It is also expressed in immune cells including macrophages, neutrophils, and dendritic cells with varying kinetics. Recent studies have shown that OPN is neuroprotective because of its role in downregulating $\operatorname{iNOS}(27,28,49)$. This decreases reactive oxygen species (ROS) generation and leads to an antiapoptotic effect by inhibiting mitochondriarelated apoptosis pathways involving cytochrome c, cleaved caspase-3, and Bax (10).

Despite the protective effect of melatonin against oxidative stress and apoptosis induced by various neurological disorders including stroke and Alzheimer's disease (AD), the effect of melatonin on SMP30 and OPN expressions in an animal model of vascular dementia (VD) has not been investigated. This study was designed to investigate the protective effect of exogenous melatonin on the expression of SMP30 and OPN, apoptosis, as well as oxidative stress parameters in a rat model of VD. Preliminary results of this study were presented in an abstract form by Ismaeel Bin-Jaliah, as a recipient of the Physiological Society Travel Grant, at the 2017 Festival of Neuroscience that was hosted in April 2017 by the British Neuroscience Association in Birmingham, England, UK.

\section{Material and Methods}

\section{Animals}

This study used 72 male Sprague-Dawley rats weighing 170-200 g. All rats were bred and housed in the research center at the Mansoura University College of Medicine (Mansoura, Egypt) at a temperature of $23 \pm 1{ }^{\circ} \mathrm{C}$ and a 12-h light-dark cycle. All rats had free access to water. They were fed standard laboratory chow during the acclimatization period before being divided into groups. All experimental procedures were approved by the medical research 
ethical committee at Mansoura University. The rats were taken care for in accordance with the Guide for the Care and Use of Laboratory Animals (1996, published by National Academy Press, 2101 Constitution Ave. NW, Washington, DC 20055, USA) (NIH publication no. 85-23, revised 1996). This work is part of the collaboration between the Department of Physiology, College of Medicine, King Khalid University, Abha, Saudi Arabia and the Department of Medical Physiology, College of Medicine, Mansoura University, Egypt.

Experimental groups and preparation of melatonin. Melatonin (Sigma, St Louis, USA) was prepared fresh every day $10 \mathrm{~min}$ before administration by dissolving $1.72 \mathrm{mg}$ of the drug in $0.5 \mathrm{ml}$ of $96 \%$ ethanol to prepare a stock solution. The working solution administered to rats was prepared by diluting $288 \mu \mathrm{l}$ of stock solution in $500 \mathrm{ml}$ drinking water to give a final melatonin concentration of $20 \mu \mathrm{g} / \mathrm{ml}$ (42). Seventy-two rats were divided into six groups ( $n=12$ each) as follows: (i) untreated control (CON), (ii) sham-operated group, (iii) sham-operated + melatonin, (iv) exposed to VD based on permanent bilateral occlusion of the common carotid arteries (BCCAO) leading to CHP was used, (v) exposed to $\mathrm{VD}+$ melatonin (BCCAO + melatonin), and (vi) exposed to $\mathrm{VD}+$ donepezil (BCCAO + DON). Melatonin was administered orally during the dark period using water bottles covered with aluminum foil. Melatonin administration started $1 \mathrm{~h}$ before induction of 2 vessel occlusion (2-VO) and continued for 28 consecutive days (36). Equal volumes of drinking water were supplied to all groups. To daily calculate melatonin consumption, the melatoninadministered groups were housed at one rat/cage, and daily water consumption was calculated. Based on this information and based on the rat's body weight, the average daily melatonin intake was $187.6 \pm 8.62 \mu \mathrm{g} / 100 \mathrm{~g}$ bwt. DON was administered intraperitoneally (i. p.) at $3 \mathrm{mg} / \mathrm{kg} /$ day for 28 consecutive days.

Global cerebral hypoperfusion (BCCAO). Permanent BCCAO with hypotension (2-VO) was used to induce CHP $(43,48)$.

Hippocampi collection. All rats were killed by cervical dislocation after being anesthetized with sodium pentobarbital (60-70 mg/kg, i.p.). Their brains were removed and immediately placed into an ice-cold dish. The hippocampus from each brain was quickly dissected according to the neuroanatomy atlas under a stereomicroscope. The hippocampus was weighed (average weight: $160 \pm 12.3 \mathrm{mg}$ ), frozen in liquid nitrogen, and stored at $-80{ }^{\circ} \mathrm{C}$ for later use. Assay of neurotransmitters, TBARS, GSH, CAT, SOD, and TAC. Hippocampus samples were transferred into a Petri dish and homogenized separately in 0.1 mol Tris-HCl buffer, $\mathrm{pH} 7.4$, using a Potter-Elvehjem homogenizer (NW Kennesaw, GA 30144, USA) at $4{ }^{\circ} \mathrm{C}$ with a diluting factor of 4 . The crude tissue homogenate was centrifuged at a speed of $9,000 \mathrm{rpm}$ for $15 \mathrm{~min}$ in a cold centrifuge. The supernatant was collected and stored at $-20{ }^{\circ} \mathrm{C}$ until the levels of dopamine (Dop), norepinephrine (NE), and acetylcholine (ACh) concentrations were measured by ELISA obtained from Abnova (Taipei City 114, Taiwan). Malondialdehyde was also measured using the thiobarbituric acid reactive substances (TBARS) method (53). Moreover, total antioxidant capacity (TAC) was measured according to a published method (20). The results are expressed as trolox equivalents per gram wet tissue weight. The levels of glutathione (GSH) (Cayman Chemical, Ann Arbor, MI, USA), superoxide dismutase (SOD) (Cayman Chemical, Ann Arbor), and catalase (CAT) (Northwest Life Science Specialties, Vancouver, WA, USA) were measured according to the manufacturer's instructions.

Immunohistochemical localization of OPN in the hippocampus. The right hippocampi of six rats from the different groups were paraffin embedded and cut into 5 - $\mu \mathrm{m}$ thick sections on a microtome. Immunohistochemistry was performed using the avidin-biotin complex (ABC) technique. First, sections were deparaffinized in xylene, dehydrated in a descending ethanol 
series $(100 \%, 90 \%$, and 70\%, v/v), immersed in a retrieval solution (DAKO 51700; DAKO Diagnostics S.A., Barcelona, Spain), and washed in phosphate-buffered solution (PBS) for 5 min. Next, the endogenous peroxidase activity was inhibited with $3 \% \mathrm{H}_{2} \mathrm{O}_{2}$ in absolute methanol for $30 \mathrm{~min}$ and washed in PBS. Non-specific antibody binding was blocked by incubating with normal goat serum (DAKO X 909; DAKO Diagnostics S.A.) in PBS diluted with the ratio of 1:4. The excess serum was blotted, and the sections were incubated with primary antibodies (rabbit anti-OPN affinity-purified polyclonal antibody) diluted with the ratio of 1:100 in PBS for $2 \mathrm{~h}$ at room temperature. Sections were washed thrice for $5 \mathrm{~min}$ in PBS. The sections were incubated for $30 \mathrm{~min}$ in pre-diluted anti-rabbit biotinylated universal secondary antibody. At the end of $30 \mathrm{~min}$, sections were washed in PBS for $5 \mathrm{~min}$ and incubated in prepared $\mathrm{ABC}$ reagent for another $30 \mathrm{~min}$ (DAKO LSAB $2 \mathrm{Kit}$; DAKO Diagnostics S.A.). Peroxidase detection was achieved using 3,30-diaminobenzidine tetrahydrochloride as a chromogen followed by washing in PBS for $5 \mathrm{~min}$. PBS was used in place of the antibody as a negative CON. Positive immunoreactions were characterized by brown dot-like cytoplasmic expression, whereas negative immunoreactivity remained unlabeled. The density of the antibody labeling was measured using an image analyzer (SAMBA 2005; Villeneuve d'Ascq, France). The density values were expressed as percentages [optical density $=\log 10$ (1/light transmission) $]$. This densitometric analysis has previously been used as a semiquantitative technique in rats (44).

Quantitative real-time PCR (Q-PCR) for determining the levels of gene expression. Total RNA isolation, purification, and concentration measurement were performed as described by Sakr et al. (41). The sequences of the primers used were designed by other authors: SMP30, forward: 5'AGGCATCAAAGTGTCTGCTGTTT3'; reverse: 5'GACTGTCGAAGTGCCA CTGAACT3' (17). Bax, forward: 5'CCAGGACGCATCCACCAAGAAGC3'; reverse: 5' TGCCACACGGAAGAAGACCTCTCG3' (136-bp product size) (23). Bcl-2, forward: 5' GGATGACTTCTCTCGTCGCTACCGT3'; reverse: 5'ATCCCTGAAGAGTTCCTCCAC CAC3' (118-bp product size) (23). $\beta$-actin, forward: 5'GTCGTACCACTGGCATTGTG3'; reverse: 5'CTCTCAGCTGTGGTGGTGAA3' (51).

Reactions were performed in duplicates as discussed previously (3). For Bax and Bcl-2, Q-PCR was performed according to the method described by Li et al. (23) using the following mix and program: the reactions were performed in a $20-\mu$ l volume mix containing $10-\mu 1$ SYBR Green I mixture, $1-\mu 1$ primers, $1-\mu \mathrm{l}$ cDNA, and $1-\mu l$ sterile, and distilled deionized water. Cycling conditions were as follows: 3 min at $95^{\circ} \mathrm{C}, 44$ cycles of $15 \mathrm{~s}$ at $95{ }^{\circ} \mathrm{C}, 20 \mathrm{~s}$ at $60{ }^{\circ} \mathrm{C}$, and $15 \mathrm{~s}$ at $72{ }^{\circ} \mathrm{C}$.

The specific amplification of each product was performed as previously described (3). The relative gene expression levels were calculated using arithmetic formulae. The CON nontreated samples were used as calibrators. The amount of target, normalized to an endogenous housekeeping gene ( $\beta$-actin) and relative to the calibrator, is given by $\Delta \Delta \mathrm{C}_{\mathrm{T}}$, where $\Delta \Delta \mathrm{C}_{\mathrm{T}}=\Delta \mathrm{C}_{\mathrm{T}}$ (sample) $-\Delta \mathrm{C}_{\mathrm{T}}$ (calibrator), and $\Delta \mathrm{C}_{\mathrm{T}}$ is the $\mathrm{C}_{\mathrm{T}}$ of the target gene subtracted from the $\mathrm{C}_{\mathrm{T}}$ of the housekeeping gene. For the untreated CON sample, $\Delta \Delta \mathrm{C}_{\mathrm{T}}$ equals zero, and $2^{0}$ equals one, so that the fold change in gene expression relative to the untreated $\mathrm{CON}$ equals one. For the treated samples (studied group samples), evaluation of $\Delta \Delta \mathrm{C}_{\mathrm{T}}$ indicates the fold change in gene expression relative to the untreated CON.

\section{Statistical analysis}

The data were expressed as the mean \pm standard deviation. Data were processed and analyzed using the SPSS version 10.0 (SPSS Inc., Chicago, IL, USA). One-way analysis of variance 
was carried out, followed by Tukey's post-hoc test. Pearson's correlation statistical analysis was performed to detect a probable significance between two different parameters. The results were considered significant, if $p \leq 0.05$.

\section{Results}

Compared with the CON group, the sham and sham + melatonin groups did not exhibit any significant changes in any of the measured parameters (data not shown).

Effect of melatonin treatment after BCCAO on the hippocampal levels of TBARS, GSH, CAT, SOD, and TAC

As expected, BCCAO produced a significant $(p<0.05)$ increase in TBARS. It also significantly decreased the TAC, SOD, CAT, and GSH $(p<0.05)$ compared with the CON group. In rats that received melatonin, TBARS significantly decreased $(p<0.05)$ with significantly increased SOD, CAT, GSH, and TAC $(p<0.05)$ compared with BCCAO in the hippocampus. Although melatonin changed TBARS, TAC, SOD, CAT, and GSH, the results were still significant compared with the CON group. DON treatment after BCCAO did not significantly change the oxidative stress parameters compared with the BCCAO and $\mathrm{BCCAO}+$ melatonin groups (Figs 1 and 2).

Effect of melatonin treatment on the hippocampal levels of $A C h, N E$, and Dop after BCCAO In response to $\mathrm{BCCAO}$, the levels of $\mathrm{ACh}, \mathrm{NE}$, and Dop significantly dropped $(p<0.05)$ in the hippocampus compared with the CON group. Melatonin treatment after BCCAO significantly $(p<0.05)$ increased the levels of ACh, NE, and Dop compared with the BCCAO group, but it was still significantly $(p<0.05)$ lower than in the CON group. Moreover, DON treatment plus BCCAO significantly $(p<0.05)$ increased the hippocampal levels of ACh, NE, and Dop compared with the BCCAO group. However, it was still
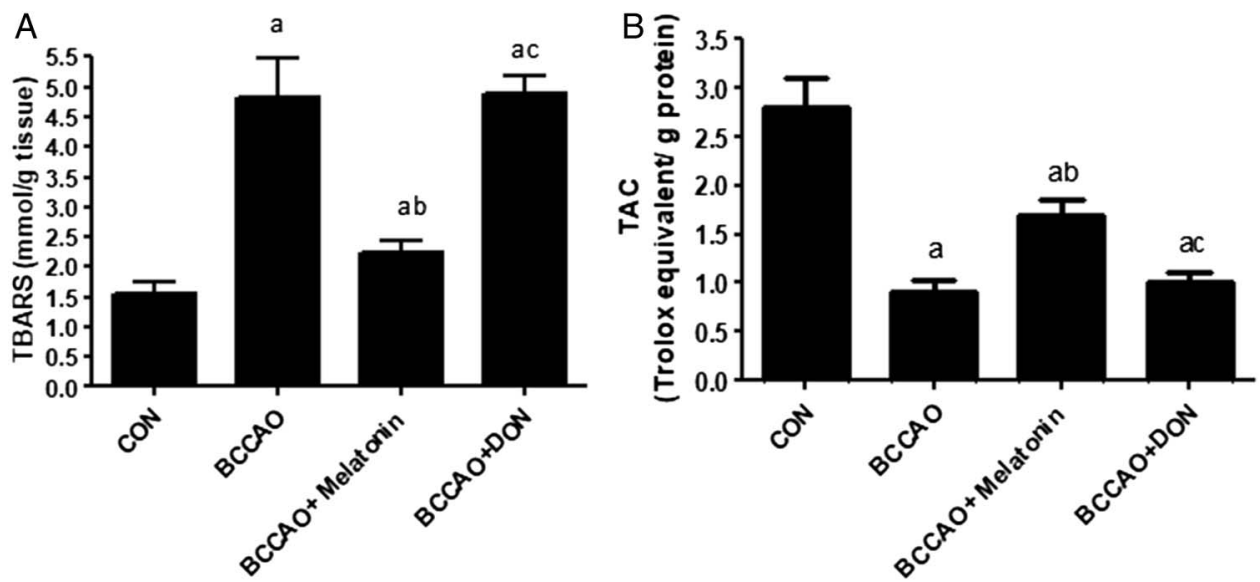

Fig. 1. The level of TBARS (A) and TAC (B) in the hippocampus. All data are expressed as the mean \pm SD $(n=12)$. CON: control group; BCCAO: bilateral common carotid artery occlusion; DON: donepezil; TBARS: thiobarbituric acid reactive substances; TAC: total antioxidant capacity. a: $p<0.05$ compared with the control group. b: $p<0.05$ compared with the BCCAO non-treated group. c: $p<0.05$ compared with the BCCAO + melatonin-treated group 

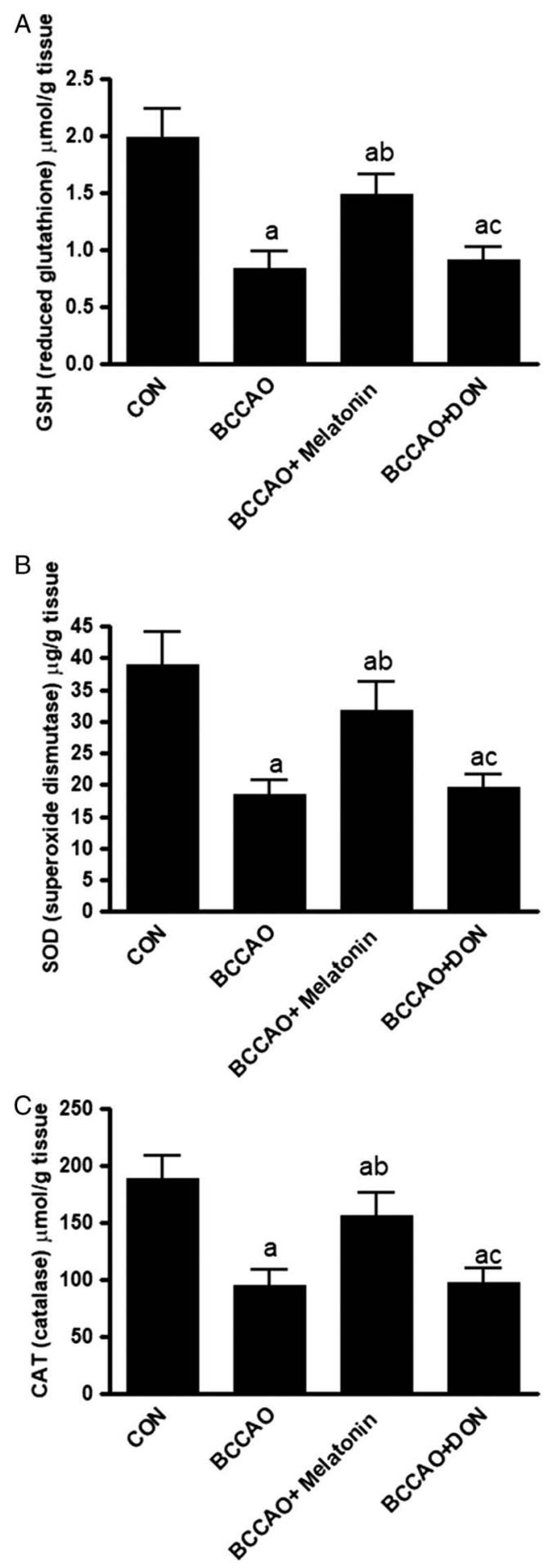

Fig. 2. The hippocampal levels of GSH (A), SOD (B), and CAT (C) hippocampus in all groups. All data are expressed as the mean $\pm \mathrm{SD}(n=12)$. a: $p<0.05$ compared with the control group. $\mathrm{b}: p<0.05$ compared with the BCCAO non-treated group. c: $p<0.05$ compared with the $\mathrm{BCCAO}+$ melatonin-treated group 
significantly $(p<0.05)$ lower than in the CON group. DON treatment after BCCAO produced an insignificant $(p>0.05)$ increased ACh compared with the BCCAO + melatonin group. On the other hand, melatonin significantly $(p<0.05)$ increased the hippocampal levels of both NE and Dop compared with the DON group (Fig. 3).

Effect of melatonin treatment on the expression of SMP30, Bax, and Bcl-2 in the rat hippocampus

Compared with CON rats, SMP30 and Bcl-2 significantly decreased $(p<0.05)$ with a significant increase $(p<0.05)$ in Bax in response to BCCAO and BCCAO + DON (Fig. 4). Melatonin increased the expression of SMP30 and Bcl-2 and downregulated Bax significantly $(p<0.05)$ compared with BCCAO. Melatonin significantly $(p<0.05)$ restored SMP30, Bcl-2, and Bax expression to levels comparable with CON. BCCAO + DON produced less ameliorative effects compared with melatonin.

Effect of melatonin treatment on the expression of OPN in the rat hippocampus

In response to BCCAO, the expression of OPN significantly decreased $(p>0.05)$ compared with that in $\mathrm{CON}$ rats (Figs 5 and 6). Melatonin after BCCAO significantly increased $(p<0.05)$ the OPN expression compared with the BCCAO group, but it was still significantly lower $(p<0.05)$ than that in the $\mathrm{CON}$ group.

\section{Correlations}

Figure 7A showed a negative Pearson's correlation between TBARS and SMP30 $(r=-0.8095)(p<0.0001)$ for all rats $(n=48)$. Figure 7B showed a negative Pearson's correlation between TBARS and OPN $(r=-0.9396)(p<0.0001)$ for all rats $(n=48)$.

\section{Discussion}

This study was performed with BCCAO to study the effect of melatonin on VD in rats. VD is associated with: (i) decreased $\mathrm{ACh}, \mathrm{NE}$, and Dop; (ii) increased oxidative stress parameters with decreased antioxidant enzymes; (iii) increased apoptosis marked by decreased Bcl-2 and increased Bax; and (iv) decreased expression of both OPN and SMP30. Treatment with the neurohormone melatonin for 28 days in a rat model of VD ameliorated oxidative stress, increased antioxidant enzymes and capacity, and upregulated the suppressed expression of OPN and SMP30. In addition, the antiapoptosis gene Bcl-2 increased with decreased Bax expression in the hippocampus.

Hippocampi isolated from BCCAO rats showed a significant decrease in the level of the neurotransmitters compared with the CON group. These results were consistent with previous studies (43). On the other hand, melatonin increased the level of neurotransmitters. These data agreed with previous studies that examined the effects of melatonin on various rat models of neurotoxicity. The mechanism by which melatonin increased ACh is dependent upon the prevention of peroxynitrite-induced inhibition of choline transport and choline acetyl transferase activity in several neuronal proteins from synaptosomes (24) and inhibition of NE and Dop reuptake (7). In this study, we used DON at $3 \mathrm{mg} / \mathrm{kg} /$ day i.p. as described by Birks and Harvey (5). They concluded that it improves cognition in dementia. In addition, Sakr et al. (43) confirmed that DON improved the working and reference memories in a rat model of VD (43). DON is one of the three ACh esterase inhibitors 

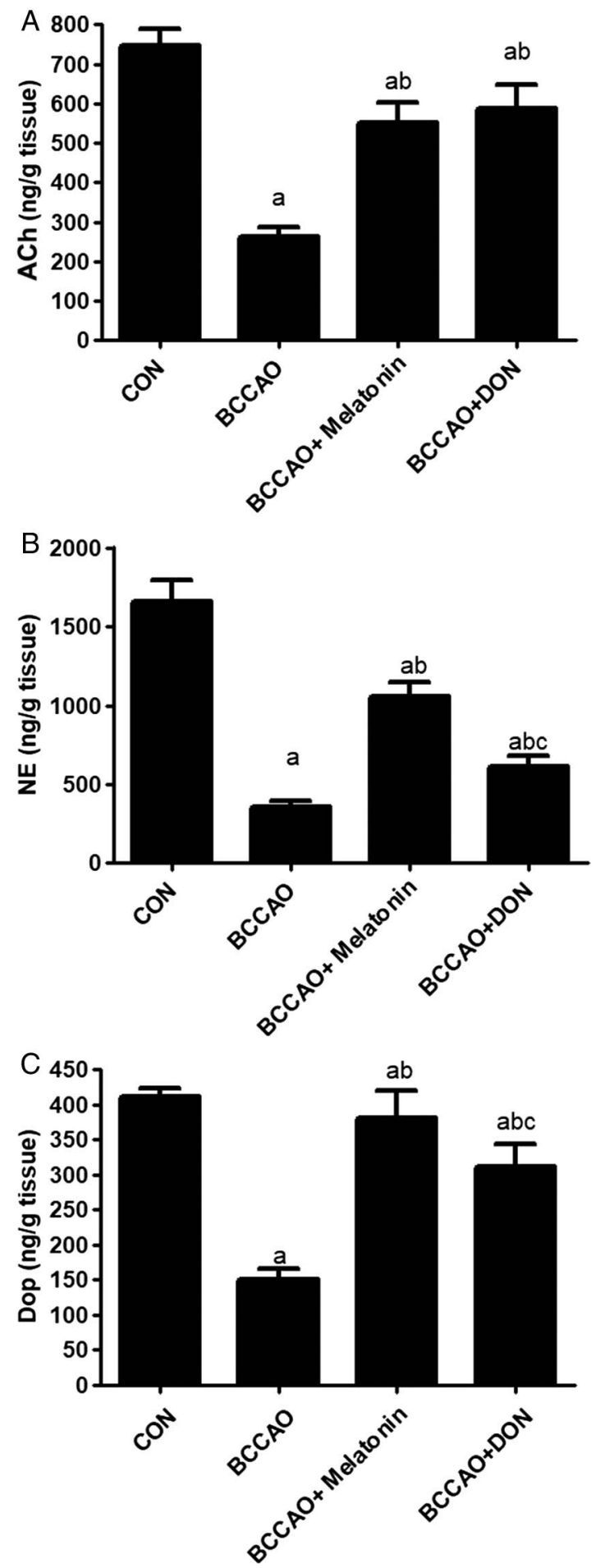

Fig. 3. The level of acetylcholine (A), norepinephrine (B), and dopamine (C) (ng/mg tissue) in the hippocampus. All data are expressed as the mean $\pm \mathrm{SD}(n=12)$. a: $p<0.05$ compared with the control group. b: $p<0.05$ compared with the BCCAO nontreated group. c: $p<0.05$ compared with the $\mathrm{BCCAO}+$ melatonin-treated group 

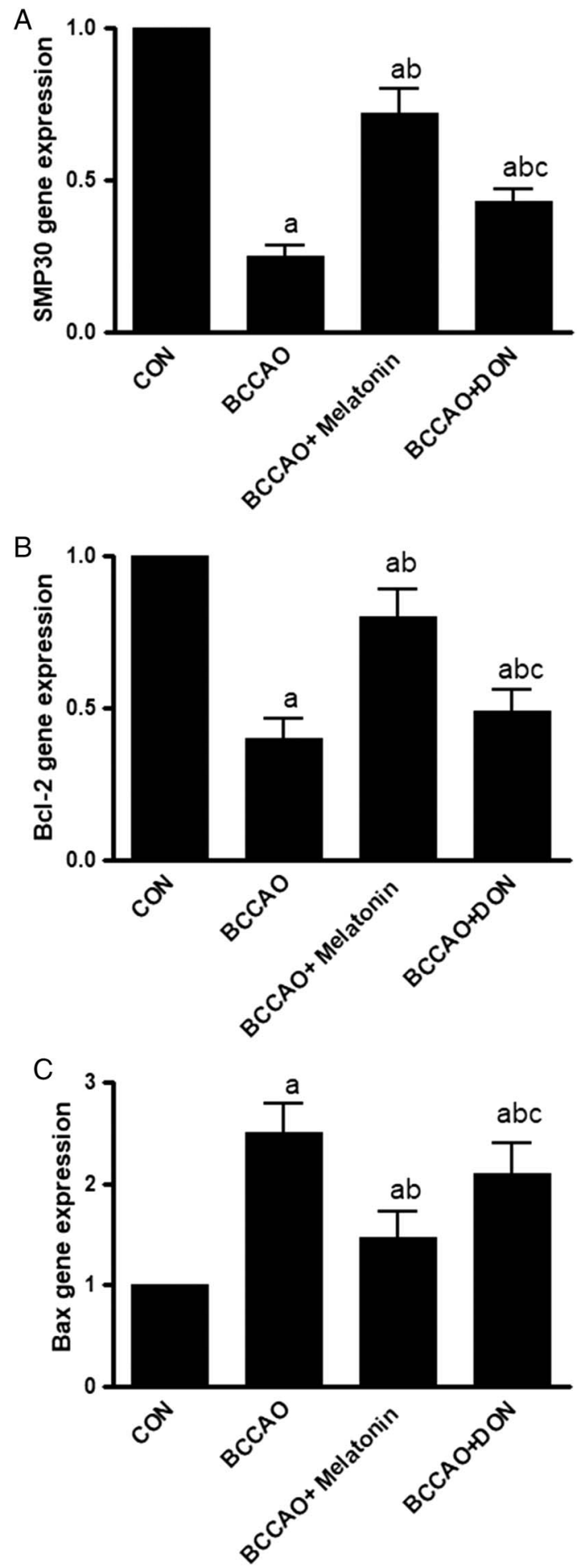

Fig. 4. (A) SMP30, (B) Bcl-2, and (C) Bax expression in the hippocampus of all groups. All data are expressed as the mean \pm SD $(n=12)$ a: $p<0.05$ compared with the control group. b: $p<0.05$ compared with the BCCAO non-treated group. c: $p<0.05$ compared with the $\mathrm{BCCAO}+$ melatonin-treated group 

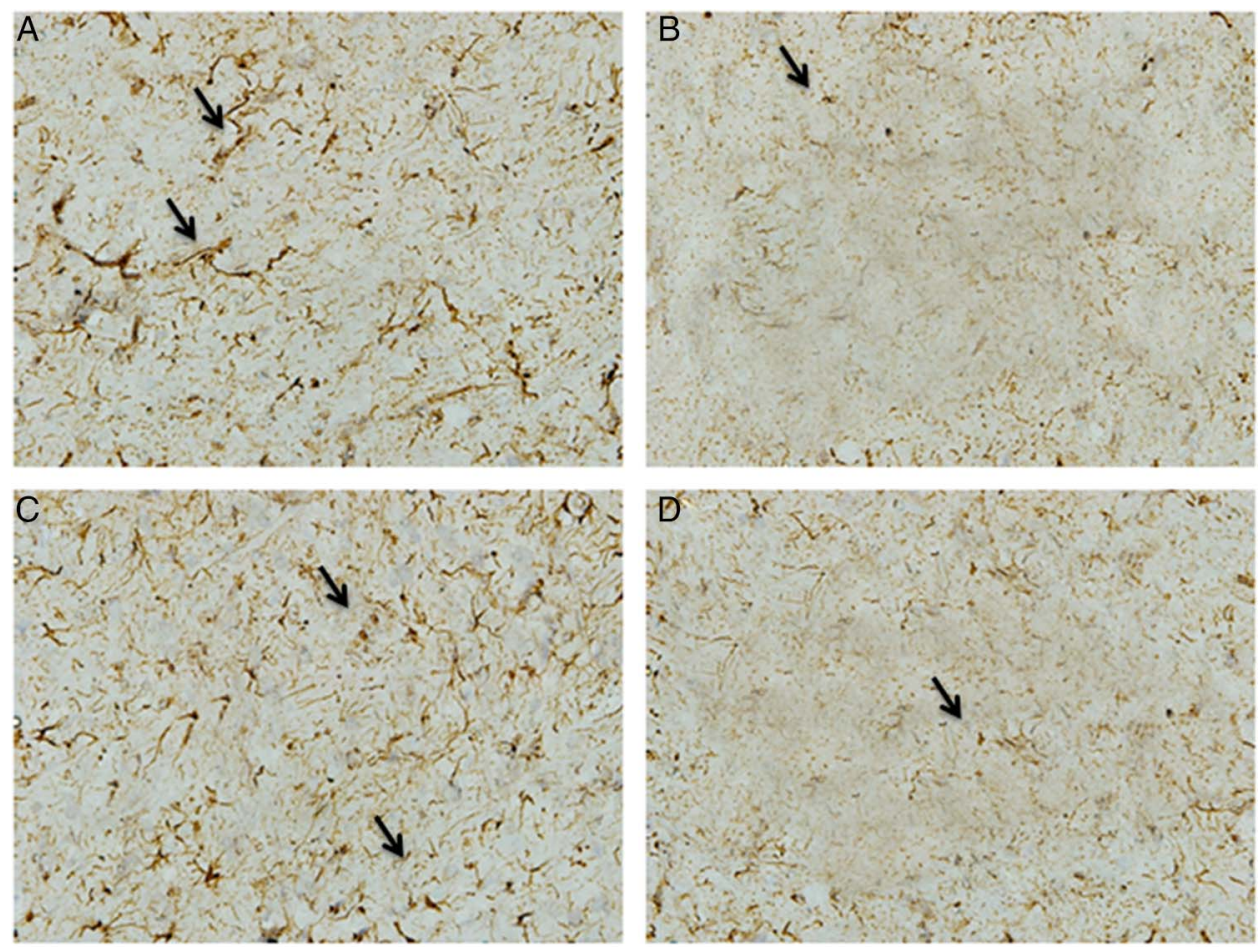

Fig. 5. Osteopontin immunoreactivity (arrows) appears as brown pigment. (A) Control group, (B) untreated BCCAO group, (C) BCCA + melatonin, (D) BCCAO + DON

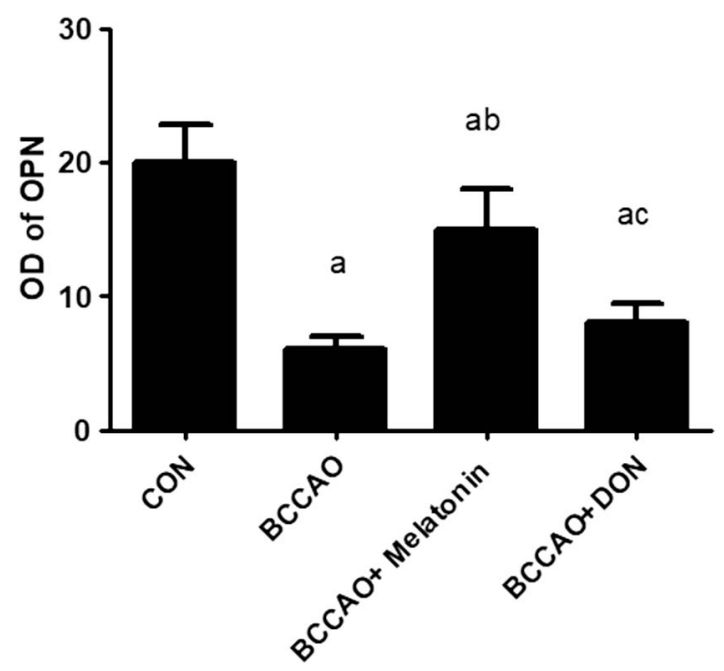

Fig. 6. Densitometric analysis of the immunohistochemical labeling of osteopontin (OPN) protein in the hippocampus tissues of all groups of rats. Values are given as the mean $\pm \mathrm{SD}$ of five areas/slide of six rats in each group. OD: optical density. a: $p<0.05$ compared with the control group. b: $p<0.05$ compared with the BCCAO non-treated group. c: $p<0.05$ compared with the $\mathrm{BCCAO}+$ melatonin-treated group

currently approved for treating $\mathrm{AD}$ symptoms and delaying the decline in cognitive function (32).

It is well known that ischemic hypoxia is associated with decreased neuronal activity, neurotransmitter synthesis, and uptake (47). BCCAO increased oxidative stress in the 

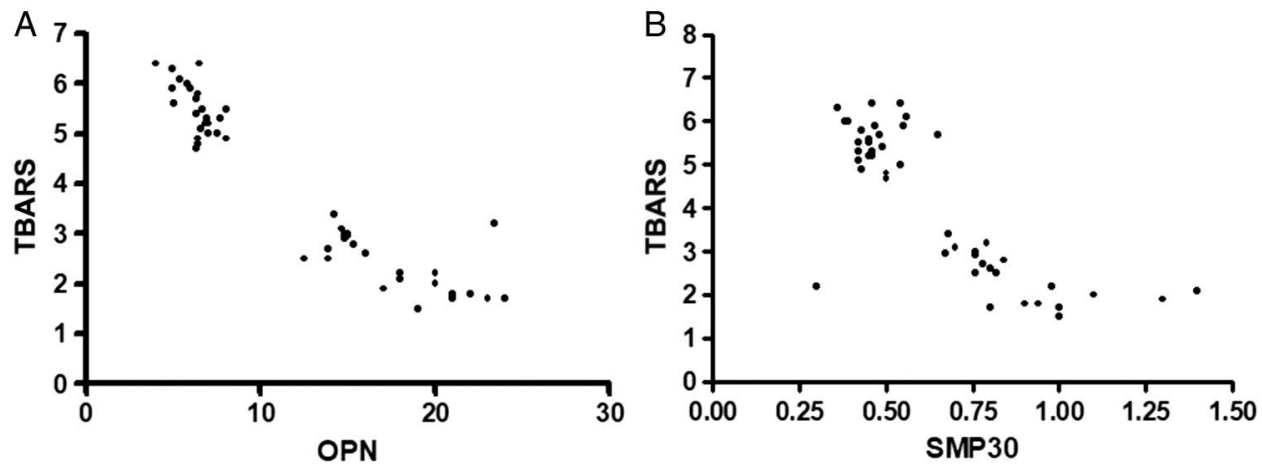

Fig. 7. (A) shows a negative correlation between TBARS and OPN $(r=-0.9396)(p<0.0001)$ for all rats $(n=48)$. (B) shows a negative correlation between TBARS and SMP30 $(r=-0.8095)(p<0.0001)$ for all rats $(n=48)$

hippocampus compared with CON rats. Our results showed that chronic ischemia increased the hippocampus TBARS and decreased GSH, CAT, and SOD compared with CON rats due to excessive ROS generation that might induce functional and structural damage of neuronal cells $(8,9,31)$. The lack of nutrients led to a state of disturbed cellular homeostasis that ended in cell death (54). Neuronal ischemia is associated with stagnant hypoxia and a decreased partial pressure of oxygen that causes a reduction in available cellular $\mathrm{O}_{2}$, which is the ultimate electron acceptor in the electron transport chain with excessive generation of superoxide anion $\left(\mathrm{O}_{2}^{-}\right)$. In turn, hydrogen peroxide $\left(\mathrm{H}_{2} \mathrm{O}_{2}\right)$ and hydroxyl radicals $\left(\mathrm{OH}^{-}\right)$ are formed. The hypoxia-induced generation of ROS can cause protein oxidation, DNA and RNA oxidation, lipid peroxidation, and neuronal dysfunction or death (6). The brain is sensitive to oxidative stress due to (i) an abundance of polyunsaturated fatty acids, (ii) deficient antioxidant defence, (iii) a high rate of $\mathrm{O}_{2}$ utilization, and (iv) a high content of transition metals, such as copper and iron, in several regions (37). Furthermore, the antioxidant and anti-inflammatory effects of melatonin cause a reduction in the production of ROS and reduced protection against mitochondrial dysfunction $(11,26,52,56)$. Our data showed that melatonin decreased TBARS and increased SOD, CAT, GSH, and TAC. Receptor-independent actions of melatonin and its metabolites relate to the ability to directly quench free radicals and non-radical toxic species. Excessive free-radical generation is notoriously destructive and kills cells secondary to massive oxidative damage. This induces cellular apoptosis or necrosis. These results agreed with previous reports (54). Recently, Ali et al. (1) showed that melatonin could produce an antioxidant effect and could modulate the RAGE/NF-KB/JNK signaling pathway in a mouse model of aging. In addition, this study showed that melatonin modulated the ratio between Bcl-2 and Bax to inhibit apoptosis. These results agreed with previous studies $(2,14,34)$.

SMP30 acts as an anti-aging agent through a $\mathrm{Ca}^{2+}$ regulator, antioxidants, and gluconolactonase, which is a key enzyme in ascorbic acid (vitamin C) biosynthesis (30). It exerts its antioxidant effect without affecting the major antioxidant enzymes, SOD, CAT, and reduced GSH peroxidase in the brain (46). In response to cerebral ischemia, the expression of SMP30 and Bcl-2 significantly decreased with increased Bax compared with CON rats. These results suggested that hypoxia and ischemia led to the apoptotic and aging processes of the neuronal tissues through acceleration and facilitation of oxidative stress. Those findings are in agreement with previous studies (45). Instead, 
melatonin treatment after BCCAO upregulated the downregulated expression of SMP30 compared with the BCCAO group. Melatonin exerted its anti-aging effect by upregulating multiple genes. Epigenetic actions of melatonin that relate to brain aging, and neurodegenerative diseases remain poorly characterized. However, chronic treatment with melatonin in the SAMP8 model could reduce oxidative stress and neurodegenerative pathways, as well as tau phosphorylation and markers of cerebral aging and neurodegeneration, in SAMP8 brains. This indicated the neuroprotective and anti-aging effect of melatonin (15). Recently, Yun et al. (55) showed that melatonin restored the expression of SMP30, which is normally suppressed by p-cresol in chronic kidney failure patients due to induction of the autophagy pathway. Their findings suggested that the administration of melatonin could rescue renal function in patients with chronic kidney failure (55).

Agreeing with our results, a previous study demonstrated that spinal cord ischemia could depress the expression of OPN (40). Several studies advocate that OPN might be involved in the cellular response to ischemic injury. Its effects in ischemia have been difficult to ascertain. OPN has actions that are both pro-inflammatory and anti-inflammatory (27). Previous studies on nervous tissues have shown that the OPN mRNA is expressed in rat's CNS neurons, and it is upregulated in microglia in response to brain (21) and spinal cord injury (16). In response to melatonin treatment, OPN significantly increased compared with BCCAO. Previous reports that concur with our data concluded that melatonin increased OPN expression and synthesis $(39,50)$. OPN increased to exert its neuroprotective effects against tissue hypoxia and ischemia by decreasing the induction of nitric oxide synthase, increasing NF-kB activity and increasing PI3K activation (28). In addition, the upregulation of OPN aids in inhibiting cleaved caspase-3 (10). OPN is inversely correlated with TBARS in these data.

These findings could suggest that melatonin exerted anti-aging and antioxidant effects by upregulating the downregulated SMP30 and OPN. Therefore, treating with natural antioxidants and free-radical scavengers such as melatonin is a possible therapeutic strategy for ameliorating oxidative stress and brain injury resulting from CHP. Further research on these molecules is required to explore their potential and confirm their candidature as neuroprotective drugs.

One of the limitations of this study was the separation of melatonin-treated rats. They were housed separately, whereas all other rats were housed in groups. Isolation might alter several physiological functions, and melatonin may modify these physiological functions.

\section{Conclusions}

CHP increased oxidative stress and reduced expression of the antiapoptotic OPN and the antiaging SMP30. Post-ischemic treatment with melatonin could exert neuroprotective effects by upregulating the suppressed anti-aging genes, antiapoptotic genes, and antioxidant enzymes.

\section{Acknowledgements}

The authors would like to express their gratitude to King Khalid University, Abha, Saudi Arabia and Mansoura University, Mansoura, Egypt for providing administrative and technical support.

\section{Conflict of interest}

The authors have no conflict of interest to declare. 


\section{REFERENCES}

1. Ali T, Badshah H, Kim TH, Kim MO: Melatonin attenuates D-galactose-induced memory impairment, neuroinflammation and neurodegeneration via RAGE/NF-K B/JNK signaling pathway in aging mouse model. J. Pineal Res. 58, 71-85 (2015)

2. Ali T, Kim MO: Melatonin ameliorates amyloid beta-induced memory deficits, tau hyperphosphorylation and neurodegeneration via PI3/Akt/GSk3beta pathway in the mouse hippocampus. J. Pineal Res. 59, 47-59 (2015)

3. Arruda LF, Arruda SF, Campos NA, de Valencia FF, Siqueira EM: Dietary iron concentration may influence aging process by altering oxidative stress in tissues of adult rats. PLoS One 8, e61058 (2013)

4. Battistin L, Cagnin A: Vascular cognitive disorder. A biological and clinical overview. Neurochem. Res. 35 , 1933-1938 (2010)

5. Birks J, Harvey RJ: Donepezil for dementia due to Alzheimer's disease. Cochrane Database Syst. Rev. 25, CD001190 (2006)

6. Butterfield DA, Lauderback CM: Lipid peroxidation and protein oxidation in Alzheimer's disease brain: potential causes and consequences involving amyloid beta-peptide-associated free radical oxidative stress. Free Radic. Biol. Med. 32, 1050-1060 (2002)

7. Cardinali DP, Nagle CA, Freire F, Rosner JM: Effects of melatonin on neurotransmitter uptake and release by synaptosome-rich homogenates of the rat hypothalamus. Neuroendocrinology 18, 72-85 (1975)

8. Chen SD, Lee JM, Yang DI, Nassief A, Hsu CY: Combination therapy for ischemic stroke: potential of neuroprotectants plus thrombolytics. Am. J. Cardiovasc. Drugs 2, 303-313 (2002)

9. Chen SD, Lin TK, Yang DI, Lee SY, Shaw FZ, Liou CW, Chuang YC: Protective effects of peroxisome proliferator-activated receptors gamma coactivator-1alpha against neuronal cell death in the hippocampal CA1 subfield after transient global ischemia. J. Neurosci. Res. 88, 605-613 (2010)

10. Chen W, Ma Q, Suzuki H, Hartman R, Tang J, Zhang JH: Osteopontin reduced hypoxia-ischemia neonatal brain injury by suppression of apoptosis in a rat pup model. Stroke 42, 764-769 (2011)

11. Das A, McDowell M, Pava MJ, Smith JA, Reiter RJ, Woodward JJ, Varma AK, Ray SK, Banik NL: The inhibition of apoptosis by melatonin in VSC4.1 motoneurons exposed to oxidative stress, glutamate excitotoxicity, or TNF-alpha toxicity involves membrane melatonin receptors. J. Pineal Res. 48, 157-169 (2010)

12. Du X, Chen L, Huang D, Peng Z, Zhao C, Zhang Y, Zhu Y, Wang Z, Li X, Liu G: Elevated apoptosis in the liver of dairy cows with ketosis. Cell. Physiol. Biochem. 43, 568-578 (2017)

13. Esteban S, Garau C, Aparicio S, Moranta D, Barcelo P, Fiol MA, Rial R: Chronic melatonin treatment and its precursor L-tryptophan improve the monoaminergic neurotransmission and related behavior in the aged rat brain. J. Pineal Res. 48, 170-177 (2010)

14. Feng Z, Cheng Y, Zhang JT: Long-term effects of melatonin or 17 beta-estradiol on improving spatial memory performance in cognitively impaired, ovariectomized adult rats. J. Pineal Res. 37, 198-206 (2004)

15. Gutierrez-Cuesta J, Tajes M, Jimenez A, Camins A, Pallas M: Effects of melatonin in the brain of the senescence-accelerated mice-prone 8 (SAMP8) model. Rev. Neurol. 52, 618-622 (2011)

16. Hashimoto M, Koda M, Ino H, Murakami M, Yamazaki M, Moriya H: Upregulation of osteopontin expression in rat spinal cord microglia after traumatic injury. J. Neurotrauma 20, 287-296 (2003)

17. Huang Q, Dunn RT 2nd, Jayadev S, DiSorbo O, Pack FD, Farr SB, Stoll RE, Blanchard KT: Assessment of cisplatin-induced nephrotoxicity by microarray technology. Toxicol. Sci. 63, 196-207 (2001)

18. Hudkins KL, Giachelli CM, Cui Y, Couser WG, Johnson RJ, Alpers CE: Osteopontin expression in fetal and mature human kidney. J. Am. Soc. Nephrol. 10, 444-457 (1999)

19. Ishigami A, Handa S, Maruyama N, Supakar PC: Nuclear localization of senescence marker protein-30, SMP30, in cultured mouse hepatocytes and its similarity to RNA polymerase. Biosci. Biotechnol. Biochem. 67, 158-160 (2003)

20. Katalinic V, Modun D, Music I, Boban M: Gender differences in antioxidant capacity of rat tissues determined by 2,2'-azinobis (3-ethylbenzothiazoline 6-sulfonate; ABTS) and ferric reducing antioxidant power (FRAP) assays. Comp. Biochem. Physiol. C Toxicol. Pharmacol. 140, 47-52 (2005)

21. Kim SY, Choi YS, Choi JS, Cha JH, Kim ON, Lee SB, Chung JW, Chun MH, Lee MY: Osteopontin in kainic acid-induced microglial reactions in the rat brain. Mol. Cells 13, 429-435 (2002)

22. Li DD, Luo Z, Chen GH, Song YF, Wei CC, Pan YX: Identification of apoptosis-related genes Bcl2 and Bax from yellow catfish Pelteobagrus fulvidraco and their transcriptional responses to waterborne and dietborne zinc exposure. Gene 633, 1-8 (2017) 
23. Li GY, Xie P, Li HY, Hao L, Xiong Q, Qiu T: Involment of p53, Bax, and Bcl-2 pathway in microcystinsinduced apoptosis in rat testis. Environ. Toxicol. 26, 111-117 (2011)

24. Malhotra S, Sawhney G, Pandhi P: The therapeutic potential of melatonin: a review of the science. MedGenMed. 6, 46 (2004)

25. Manchester LC, Coto-Montes A, Boga JA, Andersen LP, Zhou Z, Galano A, Vriend J, Tan DX, Reiter RJ: Melatonin: an ancient molecule that makes oxygen metabolically tolerable. J. Pineal Res. 59, 403-419 (2015)

26. Mauriz JL, Collado PS, Veneroso C, Reiter RJ, Gonzalez-Gallego J: A review of the molecular aspects of melatonin's anti-inflammatory actions: recent insights and new perspectives. J. Pineal Res. 54, 1-14 (2013)

27. Mazzali M, Kipari T, Ophascharoensuk V, Wesson JA, Johnson R, Hughes J: Osteopontin - a molecule for all seasons. QJM 95, 3-13 (2002)

28. Meller R, Stevens SL, Minami M, Cameron JA, King S, Rosenzweig H, Doyle K, Lessov NS, Simon RP, Stenzel-Poore MP: Neuroprotection by osteopontin in stroke. J. Cereb. Blood Flow Metab. 25, 217-225 (2005)

29. Meyer JS, Rauch G, Rauch RA, Haque A: Risk factors for cerebral hypoperfusion, mild cognitive impairment, and dementia. Neurobiol. Aging 21, 161-169 (2000)

30. Miyata M, Suzuki S, Misaka T, Shishido T, Saitoh S, Ishigami A, Kubota I, Takeishi Y: Senescence marker protein 30 has a cardio-protective role in doxorubicin-induced cardiac dysfunction. PLoS One 8, e79093 (2013)

31. Niizuma K, Endo H, Chan PH: Oxidative stress and mitochondrial dysfunction as determinants of ischemic neuronal death and survival. J. Neurochem. 1, 133-138 (2009)

32. Pakaski M, Feher A, Juhasz A, Drotos G, Fazekas OC, Kovacs J, Janka Z, Kalman J: Serum adipokine levels modified by donepezil treatment in Alzheimer's disease. J. Alzheimers Dis. 38, 371-377 (2014)

33. Parada E, Buendia I, Leon R, Negredo P, Romero A, Cuadrado A, Lopez MG, Egea J: Neuroprotective effect of melatonin against ischemia is partially mediated by alpha-7 nicotinic receptor modulation and HO-1 overexpression. J. Pineal Res. 56, 204-212 (2014)

34. Reiter RJ, Mayo JC, Tan DX, Sainz RM, Alatorre-Jimenez M, Qin L: Melatonin as an antioxidant: under promises but over delivers. J. Pineal Res. 61, 253-278 (2016)

35. Reiter RJ, Tan DX, Leon J, Kilic U, Kilic E: When melatonin gets on your nerves: its beneficial actions in experimental models of stroke. Exp. Biol. Med. 230, 104-117 (2005)

36. Reiter RJ, Tan DX, Manchester LC, Qi W: Biochemical reactivity of melatonin with reactive oxygen and nitrogen species: a review of the evidence. Cell Biochem. Biophys. 34, 237-256 (2001)

37. Romuk EB, Szczurek W, Oles M, Gabrysiak A, Skowron M, Nowak P, Birkner E: The evaluation of the changes in enzymatic antioxidant reserves and lipid peroxidation in chosen parts of the brain in an animal model of Parkinson disease. Adv. Clin. Exp. Med. 26, 953-959 (2017)

38. Rosales-Corral SA, Acuna-Castroviejo D, Coto-Montes A, Boga JA, Manchester LC, Fuentes-Broto L, Korkmaz A, Ma S, Tan DX, Reiter RJ: Alzheimer's disease: pathological mechanisms and the beneficial role of melatonin. J. Pineal Res. 52, 167-202 (2012)

39. Roth JA, Kim BG, Lin WL, Cho MI: Melatonin promotes osteoblast differentiation and bone formation. J. Biol. Chem. 274, 22041-22047 (1999)

40. Sakr HF, Abbas AM, Bin-Jaliah I: Modulation of the neurological and vascular complications by grape seed extract in a rat model of spinal cord ischemia-reperfusion injury by downregulation of both osteopontin and cyclooxygenase-2. Can. J. Physiol. Pharmacol. 94, 719-727 (2016)

41. Sakr HF, Abbas AM, Elsamanoudy AZ: Effect of valsartan on cardiac senescence and apoptosis in a rat model of cardiotoxicity. Can. J. Physiol. Pharmacol. 94, 588-598 (2016)

42. Sakr HF, Al-Ani B: Modulation of proinflammatory cytokines and leukocyte mobilization by melatonin in response to sterile tissue injury in Wistar albino rats. Saudi Med. J. 34, 470-476 (2013)

43. Sakr HF, Khalil KI, Hussein AM, Zaki MS, Eid RA, Alkhateeb M: Effect of dehydroepiandrosterone (DHEA) on memory and brain derived neurotrophic factor (BDNF) in a rat model of vascular dementia. J. Physiol. Pharmacol. 65, 41-53 (2014)

44. Santana Pereira JA, de Haan A, Wessels A, Moorman AF, Sargeant AJ: The mATPase histochemical profile of rat type IIX fibres: correlation with myosin heavy chain immunolabelling. Histochem. J. 27, 715-722 (1995)

45. Sato Y, Kajiyama S, Amano A, Kondo Y, Sasaki T, Handa S, Takahashi R, Fukui M, Hasegawa G, Nakamura N, Fujinawa H, Mori T, Ohta M, Obayashi H, Maruyama N, Ishigami A: Hydrogen-rich pure water prevents superoxide formation in brain slices of vitamin C-depleted SMP30/GNL knockout mice. Biochem. Biophys. Res. Commun. 375, 346-350 (2008)

46. Son TG, Zou Y, Jung KJ, Yu BP, Ishigami A, Maruyama N, Lee J: SMP30 deficiency causes increased oxidative stress in brain. Mech. Ageing Dev. 127, 451-457 (2006) 
47. Stasiak A, Mussur M, Unzeta M, Lazewska D, Kiec-Kononowicz K, Fogel WA: The central histamine level in rat model of vascular dementia. J. Physiol. Pharmacol. 62, 549-558 (2011)

48. Sun H, Zhang J, Zhang L, Liu H, Zhu H, Yang Y: Environmental enrichment influences BDNF and NR1 levels in the hippocampus and restores cognitive impairment in chronic cerebral hypoperfused rats. Curr. Neurovasc. Res. 7, 268-280 (2010)

49. Suzuki H, Ayer R, Sugawara T, Chen W, Sozen T, Hasegawa Y, Kanamaru K, Zhang JH: Protective effects of recombinant osteopontin on early brain injury after subarachnoid hemorrhage in rats. Crit. Care Med. 38, 612-618 (2010)

50. Tachibana R, Tatehara S, Kumasaka S, Tokuyama R, Satomura K: Effect of melatonin on human dental papilla cells. Int. J. Mol. Sci. 15, 17304-17317 (2014)

51. Wang Q, Du F, Qian ZM, Ge XH, Zhu L, Yung WH, Yang L, Ke Y: Lipopolysaccharide induces a significant increase in expression of iron regulatory hormone hepcidin in the cortex and substantia nigra in rat brain. Endocrinology 149, 3920-3925 (2008)

52. Yang Y, Jiang S, Dong Y, Fan C, Zhao L, Yang X, Li J, Di S, Yue L, Liang G, Reiter RJ, Qu Y: Melatonin prevents cell death and mitochondrial dysfunction via a SIRT1-dependent mechanism during ischemic-stroke in mice. J. Pineal Res. 58, 61-70 (2015)

53. Yoshioka T, Kawada K, Shimada T, Mori M: Lipid peroxidation in maternal and cord blood and protective mechanism against activated-oxygen toxicity in the blood. Am. J. Obstet. Gynecol. 135, 372-376 (1979)

54. Yuan J: Neuroprotective strategies targeting apoptotic and necrotic cell death for stroke. Apoptosis 14, 469-477 (2009)

55. Yun SP, Han YS, Lee JH, Kim SM, Lee SH: Melatonin rescues mesenchymal stem cells from senescence induced by the uremic toxin p-cresol via inhibiting mTOR-dependent autophagy. Biomol. Ther. (Seoul) 27, 1-10 (2017). doi:10.4062/biomolther.2017.071

56. Zhang HM, Zhang Y: Melatonin: a well-documented antioxidant with conditional pro-oxidant actions. J. Pineal Res. 57, 131-146 (2014) 